\title{
The heart as a rare colorectal cancer metastases location
}

\author{
Dawid Sigorski1,2, Małgorzata Muzolf3 ${ }^{3}$ Patryk Jancewicz ${ }^{4}$, Paweł Różanowski ${ }^{5}$
}

\footnotetext{
${ }^{1}$ Department of Oncology, Faculty of Medicine, Collegium Medicum, University of Warmia and Mazury in Olsztyn, Poland

${ }^{2}$ Department of Oncology and Immuno-Oncology, The Ministry of the Interior and Administration Hospital with Warmia and Mazury
} Oncology Centre, Olsztyn, Poland

${ }^{3}$ Departament of Internal Medicine, The Ministry of the Interior and Administration Hospital with Warmia and Mazury Oncology Center, Olsztyn, Poland ${ }^{4}$ Department of Diagnostic Imaging, Regional Specialist Hospital in Olsztyn, Olsztyn, Poland

${ }^{5}$ Department of Clinical Oncology and Immuno-Oncology, Greater Poland Cancer Centre, Poznan, Poland
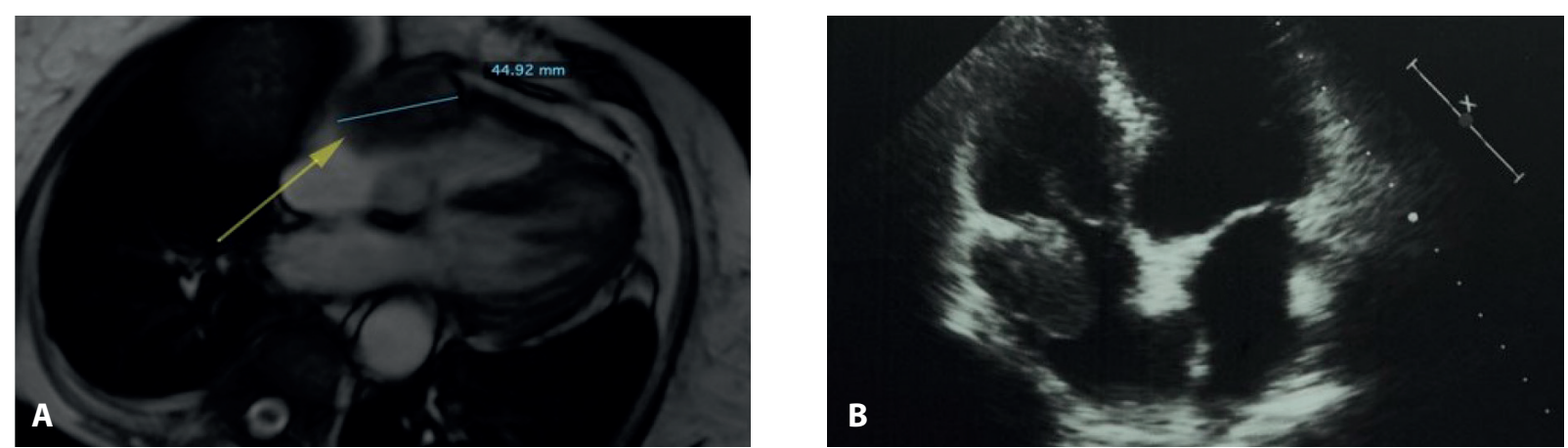

Figure 1. A. MRI: tumor mass with no signs of fat suppression seems strongly connected with the lateral and inferior wall of the right atrium, B. ECHO: right atrium tumor mass $50 \times 28 \mathrm{~mm}$ connected with the lateral wall of the atrium

The most common cardiac primary tumor is the left atrial myxoma. Secondary malignant tumors of the heart are 40-100 times more common than the primary and originate from the lungs, breast, or kidney [1, 2]. A 72-year-old woman with disseminated adenocarcinoma of the colon was admitted to the hospital. An initial hemicolectomy and ablation of liver metastases was performed. Control CT performed after 3 months showed further progression in the liver and new mass in the right atrium. She was referred to 5FU-based chemotherapy. She had no symptoms except nausea. The lab test including Troponin and NT-proBNT were in normal ranges except for the elevated CEA. The ECG was normal. Two-dimensional echocardiography (supp. video) performed routinely before chemotherapy showed a rounded mass adjacent to the wall of the right atrium. Despite chemotherapy, no regression of the cardiac tumor was observed in the echocardiography and the MRI (fig. 1). Only a few cases of colon cancer with right atrium cardiac metastases have been described in the literature. Due to the increasing incidence of malignant neoplasms, metastases to the heart will be more common. Imaging diagnostics is aimed at diagnosing and assessing the advancement of the disease.

Supp. video: Echocardiography of the right atrium mass - see on www.nowotwory.edu.pl

\section{References}

1. Akinseye OA, Ardeshna DR, Teshome MK, et al. Right ventricular metastasis from unclassified Renal Cell Carcinoma. Caspian J Intern Med. 2019; 10(2): 235-238, doi: 10.22088/cjim.10.2.235, indexed in Pubmed: 31363405.

2. Nomoto $\mathrm{N}$, Tani T, Konda T, et al. Primary and metastatic cardiac tumors: echocardiographic diagnosis, treatment and prognosis in a 15-years single center study. J Cardiothorac Surg. 2017; 12(1): 103, doi: 10.1186/ s13019-017-0672-7, indexed in Pubmed: 29183343.

\section{How to cite:}

Sigorski D, Muzolf M, Jancewicz P, Różanowski P. The heart as a rare colorectal cancer metastases location. NOWOTWORY J Oncol $2021 ; 71: 417$. 